\title{
BILINEAR MULTIPLIERS ON LORENTZ SPACES
}

\author{
FRANCISCO VILLARROYA
}

\begin{abstract}
We give one sufficient and two necessary conditions for boundedness between Lebesgue or Lorentz spaces of several classes of bilinear multiplier operators closely connected with the bilinear Hilbert transform.
\end{abstract}

\section{Introduction.}

The bilinear Hilbert transform with parameter $\alpha \in \mathbb{R}$ is the operator given by

$$
H_{\alpha}(f, g)(x)=\frac{1}{\pi} p \cdot v \cdot \int f(x-t) g(x-\alpha t) \frac{d t}{t}
$$

initially defined for functions in the Schwartz class. Notice that $H_{0}(f, g)=$ $H(f) g$ and $H_{1}(f, g)=H(f g)$ where $H(f)$ is the classical Hilbert transform. So $H_{\alpha}$ can be seen as an intermediate step between both operators.

The bilinear Hilbert transform has been extensively studied since 1965 when A. Calderón set the hypothesis of its boundedness from $L^{2} \times L^{\infty}$ into $L^{2}$ while he was working on the Hilbert transform defined over Lipschitz curves (see [2]). After several years of research and using original ideas of C. Fefferman [3], M. Lacey and C. Thiele finally answered this question when they proved the following

1991 Mathematics Subject Classification. Primary 42B10,42B15; Secondary 42B35,47H60.

Key words and phrases. Bilinear Hilbert transform, bilinear multipliers, Lorentz spaces.

The author has been partially supported by grants DGESIC PB98-1246 and BMF 2002-04013. 
Theorem 1.1. For each triple $\left(p_{1}, p_{2}, p_{3}\right)$ such that $1<p_{1}, p_{2} \leq \infty, 1 / p_{1}+$ $1 / p_{2}=1 / p_{3}$ and $p_{3}>2 / 3$ and each $\alpha \in \mathbb{R} \backslash\{0,1\}$ there exists $C\left(\alpha, p_{1}, p_{2}\right)>$ 0 for which

$$
\left\|H_{\alpha}(f, g)\right\|_{p_{3}} \leq C\left(\alpha, p_{1}, p_{2}\right)\|f\|_{p_{1}}\|g\|_{p_{2}}
$$

for all $f, g$ in the Schwartz class

in two papers ([8], [9]) published in 1997 and 1999 respectively. See also [14] for a unified proof.

From then a great deal of generalizations and extensions of this seminal work have appeared such that: [4], [5] and [12] related to the modification of the kernel of the operator, [6] related to uniform estimates in the same inequality, [10] related to maximal results, [13] to uniform estimates with generalized kernels.

The present paper shows two sufficient and one necessary conditions for boundedness of different types of bilinear multipliers some of which include the bilinear Hilbert transform.

\section{Preliminaries, notation And Definitions.}

Given a measurable function $f$ we denote its distribution function by $m_{f}(\lambda)=m(\{x \in \mathbb{R}:|f(x)|>\lambda\})$ and its nonincreasing rearrangement by $f^{*}(t)=\inf \left\{\lambda>0: m_{f}(\lambda) \leq t\right\}$. The Lorentz space $L^{p, q}$ consists of those measurable functions $f$ such that $\|f\|_{p, q}^{*}<\infty$, where

$$
\|f\|_{p, q}^{*}= \begin{cases}\left\{\frac{q}{p} \int_{0}^{\infty} t^{\frac{q}{p}} f^{*}(t)^{q} \frac{d t}{t}\right\}^{\frac{1}{q}}, & 0<p<\infty, 0<q<\infty \\ \sup _{t>0} t^{\frac{1}{p}} f^{*}(t) & 0<p \leq \infty, q=\infty\end{cases}
$$

Reader is referred to [1] for basic information on Lorentz spaces.

The interpolation result we are going to use is a trilinear version of RieszThorin interpolation theorem over tuplas of spaces. Since we will use it 
with positive integral operators

$$
\int_{\mathbb{R}} f(x-t) g(x-\alpha t) K(t) d t
$$

where $K$ is a positive function, we state the theorem in this setting.

Theorem 2.1. Let $0<p_{i, j} \leq \infty$ for $i=1, \ldots, n, j=0,1,2,3$. Let $T$ a positive trilinear integral operator such that $T: L^{p_{i, 0}} \times L^{p_{i, 1}} \times L^{p_{i, 2}} \rightarrow L^{p_{i, 3}}$ is bounded for $i=1, \ldots, n$ with $\|T\|_{i} \leq M_{i}$.

Then $T: L^{p_{0}} \times L^{p_{1}} \times L^{p_{2}} \rightarrow L^{p_{3}}$ is bounded for $\frac{1}{p_{j}}=\sum_{i=1}^{n} \frac{\theta_{i}}{p_{i, j}}$, for $j=0,1,2,3$ where $0 \leq \theta_{i} \leq 1$ and $\sum_{i=1}^{n} \theta_{i}=1$. Moreover, $\|T\| \leq \prod_{i=1}^{n} M_{i}^{\theta_{i}}$.

A proof of this theorem between pair of spaces can be seen in [1] page 185 for the linear case and 202 for the multilinear case. The extension to tuplas of spaces is trivial from that result.

We set some frequently used notation. For every $x, y \in \mathbb{R}$ we denote the translation operator by $T_{y} f(x)=f(x-y)$ and the modulation operator by $M_{y} f(x)=f(x) e^{2 \pi i y x}$ while for all $p \in \mathbb{R}$ and $t \neq 0$ we denote the dilation operators by $D_{t}^{p} f(x)=t^{-\frac{1}{p}} f\left(t^{-1} x\right)$ and $D_{t} f(x)=D_{t}^{\infty} f(x)=f\left(t^{-1} x\right)$. These operators show certain symmetries when the Fourier transform acts over them. In particular, the transform of a translation is a modulation, $\left(T_{y} f\right)^{\wedge}=$ $M_{-y} \hat{f}$, the transform of a modulation is a translation, $\left(M_{y} f\right)^{\wedge}=T_{y} \hat{f}$ and the transform of a dilation is its dual dilation, $\left(D_{t}^{p} f\right)^{\wedge}=\operatorname{sign}(t) D_{t^{-1}}^{p^{\prime}} \hat{f}$.

For the dilation operator we trivially have that $\left\|D_{t}^{r} f\right\|_{p, q}=|t|^{\frac{1}{p}-\frac{1}{r}}\|f\|_{p, q}$. Sometimes we will also use the notation $K_{\epsilon}$ for the change of scale normalized to the $L^{1}$ norm, that is, $K_{\epsilon}(x)=\epsilon^{-1} K\left(\epsilon^{-1} x\right)=D_{\epsilon}^{1} K(x)$.

The bilinear operators we are going to work with can be seen as generalizations of convolution operators. Thus, as in the case of the convolution of a distribution and a function, they can be defined functionally and distributionally. We will work only with the functional definition. 
Definition 2.1. Let $u$ be a distribution. For every $\alpha \in \mathbb{R}$ and every $f, g \in$ $C_{0}^{\infty}$ we define the function

$$
H_{u, \alpha}(f, g)(x)=\left(u, D_{-1} T_{-x} f \cdot D_{-\alpha^{-1}} T_{-x} g\right)
$$

for all $x \in \mathbb{R}$. We will say that $H_{u, \alpha}$ is a generalized bilinear Hilbert transform associated to $u$ and $\alpha$ or just a BHT for short.

In this way, if $K$ is a locally integrable function for instance this definition leads to the expression

$$
H_{K, \alpha}(f, g)(x)=\int_{\mathbb{R}} f(x-t) g(x-\alpha t) K(t) d t
$$

which is well defined for all $\alpha, x \in \mathbb{R}$ and for every $f, g$ bounded functions such that at least one of them has compact support if $\alpha \neq 0$ or $f$ has compact support if $\alpha=0$.

We give the following

Definition 2.2. Let $\alpha \in \mathbb{R}$ and $u$ be a distribution. Let $0<p_{i}<\infty$, $0<q_{i} \leq \infty, i=1,2,3$. We say that $H_{u, \alpha}$ is $\left(p_{i}, q_{i}\right)_{i=1,2,3}$ bounded if it can be extended to a bounded operator from $L^{p_{1}, q_{1}} \times L^{p_{2}, q_{2}}$ into $L^{p_{3}, q_{3}}$. This is possible if there exists a constant $C>0$ depending of $u, \alpha$ and $p_{i}, q_{i}$ such that $\left\|H_{u, \alpha}(f, g)\right\|_{p_{3}, q_{3}} \leq C\|f\|_{p_{1}, q_{1}}\|g\|_{p_{2}, q_{2}}$, for all $f$ and $g$ in some appropriate dense subspaces.

In the same way that convolution and linear multiplier operators are intimately related, so do are the operators previously defined and the following ones:

Definition 2.3. Let $m$ be a bounded measurable function in $\mathbb{R}^{2}$. For every $x \in \mathbb{R}$ and $f, g \in \mathcal{S}$ we define the operator

$$
B_{m}(f, g)(x)=\int_{\mathbb{R}^{2}} \hat{f}(\xi) \hat{g}(\eta) m(\xi, \eta) e^{2 \pi i(\xi+\eta) x} d \xi d \eta
$$


Let $p_{i}>0$. We say that $m$ is a $\left(p_{1}, p_{2}, p_{3}\right)$ multiplier or just a bilinear multiplier if the operator can be extended to a bounded operator from $L^{p_{1}} \times$ $L^{p_{2}}$ to $L^{p_{3}}$. We denote by $\|\cdot\|_{\mathcal{M} B_{p_{1}, p_{2}, p_{3}}}$ the minimum constant that satisfy the inequality $\left\|B_{m}(f, g)\right\|_{p_{3}} \leq C\|f\|_{p_{1}}\|g\|_{p_{2}}$ for all functions $f, g \in \mathcal{S}$.

The relationship between both kind of operators is the following: if $K$ is, we say, an integrable function then

$$
\int_{\mathbb{R}} f(x-t) g(x-\alpha t) K(t) d t=\int_{\mathbb{R}^{2}} \hat{f}(\xi) \hat{g}(\eta) \widehat{K}(\xi+\alpha \eta) e^{2 \pi i(\xi+\eta) x} d \xi d \eta
$$

and so, both operators can be regarded as generalization of convolution operators or as generalization of linear multiplier operators.

We finally state several of their properties related to invariance by traslation, commutativity and duality:

$$
\begin{aligned}
H_{T_{y} u, \alpha}(f, g) & =H_{u, \alpha}\left(T_{y} f, T_{\alpha y} g\right) \\
H_{u, \alpha}(f, g) & =\operatorname{sign}(\alpha) H_{D_{\alpha}^{1} u, \alpha^{-1}}(g, f) \\
\left\langle h, H_{u, \alpha}(f, g)\right\rangle & =\left\langle H_{D_{-1} u, 1-\alpha}(h, g), f\right\rangle
\end{aligned}
$$

\section{THREE CONDITIONS FOR BOUNDEDNESS}

We introduce three results of boundedness which can be summarized as follows. We first give a necessary condition obtained when we study the operator acting over gaussian functions. Then we also give a sufficient condition which is the generalization of Young inequality to this class of non-convolution operators. The third one is another sufficient condition for the second class of operators we have defined.

3.1. Gaussians looking for necessary conditions. We use the fact that the BHT over gaussian functions has a particularly easy expresion in order to get necessary conditions for its boundedness when the kernel is a temperate distribution. We get in this way two conditions of boundedness: one over 
the spaces between which the BHT can be bounded and another one over the kernel itself. We work with Lorentz spaces just for the sake of generality. We begin with a technical lemma.

Lemma 3.1. Let $G \in \mathcal{S}$ such that $\hat{G}(0)=1$. Let $\left(G_{\epsilon}\right)_{\epsilon>0}$ an approximate identity with $G_{\epsilon}=D_{\epsilon}^{1} G$. Then for all $\varphi \in \mathcal{S},\left(G_{\epsilon} * \varphi\right)_{\epsilon>0}$ converges to $\varphi$ in the topology of the Schwartz class $\mathcal{T}_{\mathcal{S}}$.

Proof. We need to prove that for every $n, m \in \mathbb{N}, \lim _{\epsilon \rightarrow 0^{+}} \|\left(G_{\epsilon} * \varphi\right)_{n, m}-$ $\varphi_{n, m} \|_{\infty}=0$ where we define $\left.\varphi_{n, m}(x)=x^{n} \varphi^{m}\right)(x)$. If $c_{n, k}$ denote the combinatorial number $n$ over $k$ then for $x \in \mathbb{R}$ and $\epsilon>0$ we have

$$
\begin{aligned}
& x^{n}\left(G_{\epsilon} * \varphi\right)^{m)}(x)=x^{n}\left(G_{\epsilon} * \varphi^{m)}\right)(x)=\int_{\mathbb{R}}(x-t+t)^{n} G_{\epsilon}(t) \varphi^{m)}(x-t) d t \\
= & \sum_{k=0}^{n} c_{n, k} \int_{\mathbb{R}} t^{k} D_{\epsilon}^{1} G(t)(x-t)^{n-k} \varphi^{m)}(x-t) d t=\sum_{k=0}^{n} c_{n, k} \epsilon^{k}\left(D_{\epsilon}^{1}\left(G_{k, 0}\right) * \varphi_{n-k, m}\right)(x)
\end{aligned}
$$

Thus,

$\left|\left(G_{\epsilon} * \varphi\right)_{n, m}(x)-\varphi_{n, m}(x)\right| \leq\left|\left(G_{\epsilon} * \varphi_{n, m}\right)(x)-\varphi_{n, m}(x)\right|+\sum_{k=1}^{n} c_{n, k} \epsilon^{k}\left\|G_{k, 0}\right\|_{1}\left\|\varphi_{n-k, m}\right\|_{\infty}$

and for $a=\max (n, m), \rho_{r}(\varphi)=\sup _{m, n \leq r}\left\|\varphi_{n, m}\right\|_{\infty}$

$\left\|\left(G_{\epsilon} * \varphi\right)_{n, m}-\varphi_{n, m}\right\|_{\infty} \leq\left\|G_{\epsilon} * \varphi_{n, m}-\varphi_{n, m}\right\|_{\infty}+\left((\epsilon+1)^{n}-1\right) \max _{0 \leq k \leq a}\left\|G_{k, 0}\right\|_{1} \rho_{a}(\varphi)$

This proves the result by the main property of an approximate identity.

Proposition 3.1. Let $\alpha<0$ and $p_{i}, q_{i}>0$ for $i=1,2,3$. Let $u$ be a non null temperated distribution. If $H_{u, \alpha}$ is bounded from $L^{p_{1}, q_{1}} \times L^{p_{2}, q_{2}}$ into $L^{p_{3}, q_{3}}$ with norm $\left\|H_{u, \alpha}\right\|$ then $0 \leq \frac{1}{p_{1}}+\frac{1}{p_{2}}-\frac{1}{p_{3}} \leq 1$.

In this case, if $G(x)=e^{-\pi x^{2}}$ and $\frac{1}{p}=\frac{1}{p_{1}}+\frac{1}{p_{2}}-\frac{1}{p_{3}}$ we have that $\hat{u} * D_{\lambda}^{p^{\prime}} G$ is a uniformly bounded family of functions with

$$
\sup _{\lambda>0}\left\|\hat{u} * D_{\lambda}^{p^{\prime}} G\right\|_{\infty} \leq C\left\|H_{u, \alpha}\right\|
$$

where $C$ is a constant that depends only of $\alpha, p_{i}$ and $q_{i}, i=1,2,3$. 
Remark 3.1. When $\frac{1}{p_{1}}+\frac{1}{p_{2}}=\frac{1}{p_{3}}$ the thesis says that $\hat{u}$ is a bounded function with $\|\hat{u}\|_{\infty} \leq C\left\|H_{u, \alpha}\right\|$ which is a known fact for linear multipliers (see [1]]).

Proof. Let $\omega \in \mathbb{R}, \lambda>0, \alpha \in \mathbb{R} \backslash\{0,1\}$ and define $\lambda^{\prime}=(1+|\alpha|)^{-1} \lambda^{2}$. Let $f(t)=e^{2 \pi i \omega t} e^{-\lambda^{\prime} \pi t^{2}}$ and $g(t)=e^{-\frac{\lambda^{\prime}}{|\alpha|} \pi t^{2}}$. An easy computation shows that for $\alpha<0$ we have $f(x-t) g(x-\alpha t)=f(x) g(x) f(-t) g(-\alpha t)$. Thus

$$
H_{u, \alpha}(f, g)(x)=f(x) g(x) H_{u, \alpha}(f, g)(0)
$$

which says that the BHT of these gaussian functions is the product of both functions times a constant. Since

$$
\left|H_{u, \alpha}(f, g)(0)\right|\|f g\|_{p_{3}, q_{3}}=\left\|H_{u, \alpha}(f, g)\right\|_{p_{3}, q_{3}} \leq\left\|H_{u, \alpha}\right\|\|f\|_{p_{1}, q_{1}}\|g\|_{p_{2}, q_{2}}
$$

we just need to compute norms in order to get the desired condition:

$$
\begin{gathered}
\|f\|_{p_{1}, q_{1}}=\left\|M_{\omega} D_{\lambda^{\prime}-1 / 2} G\right\|_{p_{1}, q_{1}}=\lambda^{\prime-\frac{1}{2 p_{1}}}\|G\|_{p_{1}, q_{1}} \\
\|g\|_{p_{2}, q_{2}}=\left\|D_{\left(\frac{\lambda^{\prime}}{\alpha \mid}\right)^{-1 / 2}} G\right\|_{p_{2}, q_{2}}=\lambda^{\prime-\frac{1}{2 p_{2}}}|\alpha|^{\frac{1}{2 p_{2}}}\|G\|_{p_{2}, q_{2}} \\
\|f g\|_{p_{3}, q_{3}}=\left\|M_{\omega} D_{\lambda^{\prime}-\frac{1}{2}\left(1+\frac{1}{|\alpha|}\right)^{-\frac{1}{2}}} G\right\|_{p_{3}, q_{3}}=\lambda^{\prime-\frac{1}{2 p_{3}}}\left(1+\frac{1}{|\alpha|}\right)^{-\frac{1}{2 p_{3}}}\|G\|_{p_{3}, q_{3}}
\end{gathered}
$$

with

$$
\|G\|_{p_{i}, q_{i}}=\left(\frac{q_{i}}{2 p_{i}}\right)^{\frac{1}{q_{i}}} \Gamma\left(\frac{q_{i}}{2 p_{i}}\right)^{\frac{1}{q_{i}}}\left(\frac{4}{q_{i} \pi}\right)^{\frac{1}{2 p_{i}}}
$$

where $\Gamma$ denotes the function Gamma of Euler (see remark 3.2 below). So

$$
\begin{gathered}
\left|H_{u, \alpha}(f, g)(0)\right| \leq\left\|H_{u, \alpha}\right\| \frac{\|G\|_{p_{1}, q_{1}}\|G\|_{p_{2}, q_{2}}}{\|G\|_{p_{3}, q_{3}}}|\alpha|^{\frac{1}{2 p_{2}}}\left(1+\frac{1}{|\alpha|}\right)^{\frac{1}{2 p_{3}}} \lambda^{\prime-\frac{1}{2}\left(\frac{1}{p_{1}}+\frac{1}{p_{2}}-\frac{1}{p_{3}}\right)} \\
=\left\|H_{u, \alpha}\right\| \frac{\|G\|_{p_{1}, q_{1}}\|G\|_{p_{2}, q_{2}}}{\|G\|_{p_{3}, q_{3}}}|\alpha|^{\frac{1}{2}\left(\frac{1}{p_{2}}-\frac{1}{p_{3}}\right)}(1+|\alpha|)^{\frac{1}{2}\left(\frac{1}{p_{1}}+\frac{1}{p_{2}}\right)} \lambda^{-\frac{1}{p}}=C \lambda^{-\frac{1}{p}}
\end{gathered}
$$

for all $\lambda>0$ and $\omega \in \mathbb{R}$.

Now we work a little bit the expression $H_{u, \alpha}(f, g)(0)$. Since

$$
f(-t) g(-\alpha t)=e^{-2 \pi i \omega t} e^{-(1+|\alpha|) \lambda^{\prime} \pi t^{2}}=e^{-2 \pi i \omega t} e^{-\lambda^{2} \pi t^{2}}=M_{-\omega} D_{\lambda^{-1}} G(t)
$$


we have, using the fact that $\widehat{G}=G$ and $D_{-1} G=G$, that

$$
H_{u, \alpha}(f, g)(0)=\left(u, M_{-\omega} D_{\lambda^{-1}} G\right)=\left(\hat{u}, T_{\omega} D_{\lambda}^{1} G\right)=\left(\hat{u} * D_{\lambda}^{1} G\right)(\omega)
$$

and we can rewrite the previous result for all $\lambda>0$ and $\omega \in \mathbb{R}$ as

$$
\left|\left(\hat{u} * D_{\lambda}^{1} G\right)(\omega)\right| \leq C \lambda^{-\frac{1}{p}}
$$

a) If $\frac{1}{p}<0$ we prove that $u \equiv 0$ by showing that the family of functions $m_{\lambda}(\omega)=\left(\hat{u} * D_{\lambda}^{1} G\right)(\omega)$ converge pointwise to zero and distributionally to $\hat{u}$ when $\lambda$ tends to zero.

On one side, we have $m_{\lambda}$ are bounded functions (and so locally integrable) with $\left\|m_{\lambda}\right\|_{\infty} \leq C \lambda^{-\frac{1}{p}} \leq C$ for $\lambda<1$ and $\lim _{\lambda \rightarrow 0} m_{\lambda}(\omega)=0$ for all $\omega \in \mathbb{R}$.

On the other side, since $\left(D_{\lambda}^{1} G\right)_{\lambda>0}$ is an approximate identity we have proven in lemma 3.1 that $\left.\left\{D_{\lambda}^{1} G * \varphi\right)\right\}_{\lambda>0}$ converges to $\varphi$ in the topology $\mathcal{T}_{\mathcal{S}}$. Thus, by continuity of $\hat{u}$ we have for all $\varphi \in \mathcal{S}$

$$
\lim _{\lambda \rightarrow 0}\left(u_{m_{\lambda}}, \varphi\right)=\lim _{\lambda \rightarrow 0}\left(\hat{u} * D_{\lambda}^{1} G, \varphi\right)=\lim _{\lambda \rightarrow 0}\left(\hat{u}, D_{\lambda}^{1} G * \varphi\right)=(\hat{u}, \varphi)
$$

With both facts and dominated convergence theorem of Lebesgue we have

$$
(\hat{u}, \varphi)=\lim _{\lambda \rightarrow 0}\left(u_{m_{\lambda}}, \varphi\right)=\lim _{\lambda \rightarrow 0} \int_{\mathbb{R}} m_{\lambda}(\omega) \varphi(\omega) d \omega=0
$$

b) If $\frac{1}{p}=0$ we still know that $m_{\lambda}$ define a family of bounded functions with $\left\|m_{\lambda}\right\|_{\infty} \leq C$ for all $\lambda>0$ that converge distributionally to $\hat{u}$ when $\lambda$ tends to zero. We use this fact to show that $\hat{u}$ must be a bounded function and that, actually, the convergence is also pointwise. From above,

$$
|(\hat{u}, \varphi)|=\lim _{\lambda \rightarrow 0}\left|\int_{\mathbb{R}} m_{\lambda}(\omega) \varphi(\omega) d \omega\right| \leq \varlimsup_{\lambda \rightarrow 0}\left\|m_{\lambda}\right\|_{\infty}\|\varphi\|_{1} \leq C\|\varphi\|_{1}
$$

for all $\varphi \in \mathcal{S}$ and thus $\hat{u}$ is a distribution associated to a bounded function. Moreover, by property of approximate identity, we have that

$$
\lim _{\lambda \rightarrow 0} m_{\lambda}(\omega)=\lim _{\lambda \rightarrow 0}\left(\hat{u} * D_{\lambda}^{1} G\right)(\omega)=\hat{u}(\omega)
$$

almost everywhere (in all Lebesgue points of $\hat{u}$ ). 
c) If $0<\frac{1}{p} \leq 1$ our condition says that $\left|\left(\hat{u} * D_{\lambda}^{p^{\prime}} G\right)(\omega)\right| \leq C$ for all $\lambda>0$ and $\omega \in \mathbb{R}$ which is the main statement of the proposition.

We still have that $m_{\lambda}=\hat{u} * D_{\lambda}^{1} G$ define a family of bounded functions that converge distributionally to $\hat{u}$ and satisfies $\left\|m_{\lambda}\right\|_{\infty} \leq C \lambda^{-\frac{1}{p}}$ for all $\lambda>0$.

d) If $1<\frac{1}{p}$ we prove directly that $u \equiv 0$. The previous condition can be written as $\left|\left(\hat{u} * D_{\lambda} G\right)(\omega)\right| \leq C \lambda^{\frac{1}{p^{\prime}}}$ with $p^{\prime}<0$. Moreover, since $H_{u, \alpha}$ is bounded and translation invariant by property (2) , we have that $H_{T_{y} u, \alpha}$ is also a bounded operator with the same constant and thus it satisfies $\left|\left(\widehat{T_{y} u} * D_{\lambda} G\right)(\omega)\right| \leq C \lambda^{\frac{1}{p^{\prime}}}$ for every $y, \omega \in \mathbb{R}$. With this we can write

$$
\begin{aligned}
& \lim _{\lambda \rightarrow 0}\left|\left(u, T_{y} D_{\lambda}^{1} G\right)\right|=\lim _{\lambda \rightarrow 0}\left|\left(\widehat{T_{-y} u}, D_{\lambda^{-1}} G\right)\right| \\
= & \lim _{\lambda \rightarrow 0}\left|\left(\widehat{T_{-y} u} * D_{\lambda^{-1}} G\right)(0)\right| \leq \lim _{\lambda \rightarrow 0} C \lambda^{-\frac{1}{p^{\prime}}}=0
\end{aligned}
$$

Thus for every $\varphi \in \mathcal{S}$ we have by the dominated convergence theorem

$$
(u, \varphi)=\lim _{\lambda \rightarrow 0}\left(u, \varphi * D_{\lambda}^{1} G\right)=\lim _{\lambda \rightarrow 0} \int_{\mathbb{R}} \varphi(y)\left(u, T_{y} D_{\lambda}^{1} G\right) d y=0
$$

Now we see the case when $\alpha>0$. If $\alpha>1$ and $p_{3} \geq 1$ the duality formula (4) with $1-\alpha<0$ let us to apply the former result to $H_{D_{-1} u, 1-\alpha}$ in the following way: if $f, g, h$ are some properly chosen gaussian functions then

$$
\left\langle h, H_{u, \alpha}(f, g)\right\rangle=\left\langle H_{D_{-1} u, 1-\alpha}(h, g), f\right\rangle=H_{D_{-1} u, 1-\alpha}(h, g)(0)\langle h g, f\rangle
$$

which, if we claim the operator bounded, implies

$$
\left|H_{D_{-1} u, 1-\alpha}(h, g)(0)\right| \leq\left\|H_{u, \alpha}\right\| \frac{\|f\|_{p_{1}, q_{1}}\|g\|_{p_{2}, q_{2}}\|h\|_{p_{3}^{\prime}, q_{3}^{\prime}}}{|\langle h g, f\rangle|}=C \lambda^{-\frac{1}{p}}
$$

Thus by (5) and using $D_{-1}(f * g)=D_{-1} f * D_{-1} g, D_{-1} \hat{u}=\widehat{D_{-1} u}$ we have

$$
\left|\left(\hat{u} * D_{\lambda}^{1} G\right)(-\omega)\right|=\left|\left(\widehat{D_{-1} u} * D_{\lambda}^{1} G\right)(\omega)\right|=\left|H_{D_{-1} u, 1-\alpha}(h, g)(0)\right| \leq C \lambda^{-\frac{1}{p}}
$$

From here the same ideas lead to the same conclusion. 
Finally, if $0<\alpha<1$ and $p_{3} \geq 1$, the commutativity formula (3) with $\alpha^{-1}>1$ and the duality formula (4) with $1-\alpha^{-1}<0$ let us apply the same ideas to $H_{D_{-1} D_{\alpha}^{1} u, 1-\alpha^{-1}}$ to get the same conclusion:

$$
\begin{gathered}
\left\langle h, H_{u, \alpha}(f, g)\right\rangle=\left\langle h, H_{D_{\alpha}^{1} u, \alpha^{-1}}(g, f)\right\rangle \\
=\left\langle H_{D_{-1} D_{\alpha}^{1} u, 1-\alpha^{-1}}(h, f), g\right\rangle=H_{D_{-1} D_{\alpha}^{1} u, 1-\alpha^{-1}}(h, f)(0)\langle h f, g\rangle
\end{gathered}
$$

which implies

$$
\left|H_{D_{-1} D_{\alpha}^{1} u, 1-\alpha^{-1}}(h, f)(0)\right| \leq\left\|H_{u, \alpha}\right\| \frac{\|f\|_{p_{1}, q_{1}}\|g\|_{p_{2}, q_{2}}\|h\|_{p_{3}^{\prime}, q_{3}^{\prime}}}{|\langle h f, g\rangle|}=C \lambda^{-\frac{1}{p}}
$$

Now, using that $D_{\alpha}(f * g)=D_{\alpha}^{q} f * D_{\alpha}^{q^{\prime}} g$ we get by (15)

$$
\begin{gathered}
\left|\left(\hat{u} * D_{\alpha \lambda}^{1} G\right)(-\alpha \omega)\right|=\left|D_{\alpha^{-1}}\left(\hat{u} * D_{\alpha}^{1} D_{\lambda}^{1} G\right)(-\omega)\right|=\left|\left(D_{\alpha^{-1}} \hat{u} * D_{\lambda}^{1} G\right)(-\omega)\right| \\
\left.=\mid \widehat{\left(D_{-1} D_{\alpha}^{1}\right.} u * D_{\lambda}^{1} G\right)(\omega)|=| H_{D_{-1} D_{\alpha}^{1} u, 1-\alpha^{-1}}(h, f)(0) \mid \leq C \lambda^{-\frac{1}{p}}
\end{gathered}
$$

and we finish with the same ideas as before.

Remark 3.2. Since $G$ is even and non-increasing in $[0, \infty)$, we know that $G^{*}=D_{2} G$ and so we can compute $\|G\|_{p_{1}, q_{1}}^{q_{1}}$ as follows

$\frac{q_{1}}{p_{1}} \int_{0}^{\infty} t^{\frac{q_{1}}{p_{1}}} e^{-\frac{1}{4} q_{1} \pi t^{2}} \frac{d t}{t}=\frac{q_{1}}{2 p_{1}}\left(\frac{4}{q_{1} \pi}\right)^{\frac{q_{1}}{2 p_{1}}} \int_{0}^{\infty} t^{\frac{q_{1}}{2 p_{1}}} e^{-t} \frac{d t}{t}=\frac{q_{1}}{2 p_{1}}\left(\frac{4}{q_{1} \pi}\right)^{\frac{q_{1}}{2 p_{1}}} \Gamma\left(\frac{q_{1}}{2 p_{1}}\right)$

3.2. Bilinear Young inequality. The next result is the generalization of Young inequality to our bilinear non-convolution operators. We pay now special attention to the dependence of the constants from the parameter $\alpha$. In order to deal with a more general and symmetric operator, we change a little bit its definition. For the next proposition we call BHT to

$$
H_{K, \alpha, \beta}(f, g)(x)=\int_{\mathbb{R}} f(x-\alpha t) g(x-\beta t) K(t) d t
$$

defined for all $\alpha, \beta, x \in \mathbb{R}$ and $f, g \in \mathcal{S}$. 
BILINEAR MULTIPLIERS ON LORENTZ SPACES

Proposition 3.2. (Bilinear Young inequality). Let $p_{0} \geq 1$. If $K \in L^{p_{0}}$ then $H_{K, \alpha, \beta}$ is a bounded operator from $L^{p_{1}} \times L^{p_{2}}$ to $L^{p_{3}}$ with $p_{i} \geq 1$ for $i=1,2,3$ and $p_{1}^{-1}+p_{2}^{-1}+p_{0}^{-1}=1+p_{3}^{-1}$, and all $\alpha, \beta \in \mathbb{R} \backslash\{0\}$ such that $\alpha \neq \beta$. Moreover,

$$
\left\|H_{K, \alpha, \beta}(f, g)\right\|_{p_{3}} \leq C_{\alpha, \beta, p_{0}, p_{1}, p_{2}}\|K\|_{p_{0}}\|f\|_{p_{1}}\|g\|_{p_{2}}
$$

Remark 3.3. Notice that $p_{1}^{-1}+p_{2}^{-1}-p_{3}^{-1}=p_{0}^{\prime-1} \in[0,1]$ as proposition 3.1 says it must be. See also that this condition can be rewritten as $p_{1}^{-1}+p_{2}^{-1}+$ ${p_{3}^{\prime-1}}^{-1}=1+p_{0}^{\prime-1}$ and so one can think the point $\left(p_{1}^{-1}, p_{2}^{-1}, p_{3}^{\prime-1}\right) \in \mathbb{R}^{3}$ belongs to the plane $x+y+z=1+p_{0}^{\prime-1}$ with $1+p_{0}^{\prime-1} \in[1,2]$.

Proof. Let $p \geq 1, f, g, h, K \in \mathcal{S}$ and $I=\mid \int_{\mathbb{R}} h(x) \int_{\mathbb{R}} f(x-\alpha t) g(x-$ $\beta t) K(t) d t d x \mid$. We denote here $f_{a, b}(x, t)=f(a x+b t)$. By Hölder inequality and some changes of variables

$I \leq\left\|f_{1,-\alpha} g_{1,-\beta}\right\|_{L^{p}\left(\mathbb{R}^{2}\right)}\left\|K_{0,1} h_{1,0}\right\|_{L^{p^{\prime}\left(\mathbb{R}^{2}\right)}}=|\alpha-\beta|^{-\frac{1}{p}}\|f\|_{p}\|g\|_{p}\|K\|_{p^{\prime}}\|h\|_{p^{\prime}}$ i.e.

$$
\left\|H_{K, \alpha, \beta}(f, g)\right\|_{p} \leq|\alpha-\beta|^{-\frac{1}{p}}\|f\|_{p}\|g\|_{p}\|K\|_{p^{\prime}}
$$

$I \leq\left\|K_{0,1} g_{1,-\beta}\right\|_{L^{p}\left(\mathbb{R}^{2}\right)}\left\|f_{1,-\alpha} h_{1,0}\right\|_{L^{p^{\prime}\left(\mathbb{R}^{2}\right)}}=|\alpha|^{-\frac{1}{p^{\prime}}}\|f\|_{p^{\prime}}\|g\|_{p}\|K\|_{p}\|h\|_{p^{\prime}}$ i.e.

$$
\left\|H_{K, \alpha, \beta}(f, g)\right\|_{p} \leq|\alpha|^{-\frac{1}{p^{\prime}}}\|f\|_{p^{\prime}}\|g\|_{p}\|K\|_{p}
$$

$I \leq\left\|f_{1,-\alpha} K_{0,1}\right\|_{L^{p}\left(\mathbb{R}^{2}\right)}\left\|g_{1,-\beta} h_{1,0}\right\|_{L^{p^{\prime}\left(\mathbb{R}^{2}\right)}}=|\beta|^{-\frac{1}{p^{\prime}}}\|f\|_{p}\|g\|_{p^{\prime}}\|K\|_{p}\|h\|_{p^{\prime}}$ i.e.

$$
\left\|H_{K, \alpha, \beta}(f, g)\right\|_{p} \leq|\beta|^{-\frac{1}{p^{\prime}}}\|f\|_{p}\|g\|_{p^{\prime}}\|K\|_{p}
$$

We associate each bound of the operator from $L^{p_{1}} \times L^{p_{2}}$ to $L^{p_{3}}$ to the point $\left(p_{1}^{-1}, p_{2}^{-1}, p_{3}^{\prime-1}\right) \in \mathbb{R}^{3}$ in the plane $x+y+z=1+p^{-1}$. In this way and taking the values $p=1$ and $p=\infty$ in each of the three previous inequalities we consider the extremal points $(1,1,0),(0,0,1)$ (from the first one), $(0,1,0)$, $(1,0,1)$ (from the second), $(1,0,0)$ and $(0,1,1)$ (from the third). In this way, by using trilinear interpolation between two spaces iteratively we get 
the bounds on the surface of the convex hull of the previous six points, that is, on the surface of the octahedron drawn in the following diagram

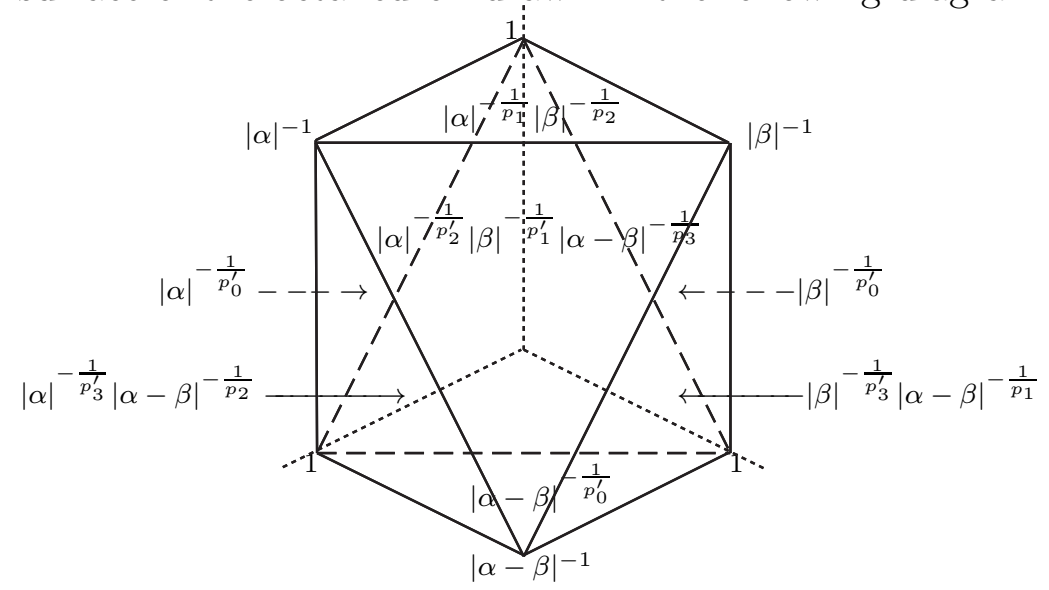

where we write the constants of boundedness in each vertex and each face.

We show how to get one of them: from (77) and (8) we know $\left\|H_{K, \alpha, \beta}(f, g)\right\|_{\infty} \leq$ $|\alpha|^{-1}\|f\|_{1}\|g\|_{\infty}\|K\|_{\infty},\left\|H_{K, \alpha, \beta}(f, g)\right\|_{\infty} \leq|\beta|^{-1}\|f\|_{\infty}\|g\|_{1}\|K\|_{\infty}$ and so we have $\left\|H_{K, \alpha, \beta}(f, g)\right\|_{\infty} \leq|\alpha|^{-\frac{1}{p}}|\beta|^{-\frac{1}{p^{\prime}}}\|f\|_{p}\|g\|_{p^{\prime}}\|K\|_{\infty}$. In the same way, from (17) and (6) $\left\|H_{K, \alpha, \beta}(f, g)\right\|_{\infty} \leq|\alpha|^{-1}\|f\|_{1}\|g\|_{\infty}\|K\|_{\infty},\left\|H_{K, \alpha, \beta}(f, g)\right\|_{\infty} \leq$ $\|f\|_{\infty}\|g\|_{\infty}\|K\|_{1}$ we get $\left\|H_{K, \alpha, \beta}(f, g)\right\|_{\infty} \leq|\alpha|^{-\frac{1}{p}}\|f\|_{p}\|g\|_{\infty}\|K\|_{p^{\prime}}$.

Interpolating both cases we get $\left\|H_{K, \alpha, \beta}(f, g)\right\|_{\infty} \leq|\alpha|^{-\frac{1}{p}}|\beta|^{-\frac{1}{q_{1}}}\|f\|_{p}\|g\|_{q_{1}}\|K\|_{q_{2}}$ with $q_{1}^{-1}+q_{2}^{-1}=p^{\prime-1}$. Using again (6) $\left\|H_{K, \alpha, \beta}(f, g)\right\|_{1} \leq|\alpha-\beta|^{-1}\|f\|_{1}\|g\|_{1}\|K\|_{\infty}$, we finally have

$$
\left\|H_{K, \alpha, \beta}(f, g)\right\|_{p_{3}} \leq|\alpha|^{-\frac{1}{p_{1}}}|\beta|^{-\frac{1}{p_{2}}}|\alpha-\beta|^{-\frac{1}{p_{3}}}\|f\|_{p_{1}}\|g\|_{p_{2}}\|K\|_{p_{0}}
$$

where $p_{3}^{-1}=\theta, p_{1}^{-1}=(1-\theta) p^{-1}+\theta, p_{2}^{-1}=(1-\theta) q_{1}^{-1}+\theta$ and $p_{0}^{-1}=$ $(1-\theta) q_{2}^{-1}$, which is the stated result since $p_{1}^{-1}+p_{2}^{-1}+p_{0}^{-1}=1+p_{3}^{-1}$.

Now in order to get bounds in the interior of the octahedron we use interpolation between six spaces. In this way, each point $p=\left(p_{1}^{-1}, p_{2}^{-1}, p_{3}^{\prime-1}\right)$ can be written as the convex linear combination of the six vertex in the following way

$$
p=\left(\lambda_{2}+p_{3}^{-1}-p_{2}^{-1}\right)(1,0,0)+\left(\lambda_{1}+p_{3}^{-1}-p_{1}^{-1}\right)(0,1,0)+\left(p_{3}^{\prime-1}-\lambda_{1}-\lambda_{2}\right)(0,0,1)
$$




$$
+\lambda_{1}(1,0,1)+\lambda_{2}(0,1,1)+\left(p_{1}^{-1}+p_{2}^{-1}-p_{3}^{-1}-\lambda_{1}-\lambda_{2}\right)(1,1,0)
$$

for every $\lambda_{1}, \lambda_{2} \in[0,1]$ such that $\max \left(p_{1}^{-1}-p_{3}^{-1}, 0\right) \leq \lambda_{1}, \max \left(p_{2}^{-1}-p_{3}^{-1}, 0\right) \leq$ $\lambda_{2}$ and $\lambda_{1}+\lambda_{2} \leq \min \left(p_{3}^{\prime-1}, p_{0}^{\prime-1}\right)$. We denote by $D$ such non empty triangle (notice that $p_{i}^{-1}-p_{3}^{-1} \leq p_{1}^{-1}+p_{2}^{-1}-p_{3}^{-1}=p_{0}^{\prime-1} \leq 1$ and $\max \left(p_{1}^{-1}-p_{3}^{-1}, 0\right)+$ $\left.\max \left(p_{2}^{-1}-p_{3}^{-1}, 0\right) \leq \min \left(p_{3}^{\prime-1}, p_{0}^{\prime-1}\right)\right)$. Also notice that this decomposition implies this other one for $\tilde{p}=\left(p_{1}^{-1}, p_{2}^{-1}, p_{3}^{-1}\right)$

$$
\begin{gathered}
\tilde{p}=\left(\lambda_{2}+p_{3}^{-1}-p_{2}^{-1}\right)(1,0,1)+\left(\lambda_{1}+p_{3}^{-1}-p_{1}^{-1}\right)(0,1,1)+\left(1-p_{3}^{\prime-1}+\lambda_{1}+\lambda_{2}\right)(0,0,0) \\
+\lambda_{1}(1,0,0)+\lambda_{2}(0,1,0)+\left(p_{1}^{-1}+p_{2}^{-1}-p_{3}^{-1}-\lambda_{1}-\lambda_{2}\right)(1,1,1)
\end{gathered}
$$

in order to interpolate. So, using theorem 2.1 we get

$$
\left\|H_{K, \alpha, \beta}(f, g)\right\|_{p_{3}} \leq|\alpha|^{-\lambda_{1}}|\beta|^{-\lambda_{2}}|\alpha-\beta|^{-\left(\frac{1}{p_{0}^{\prime}}-\lambda_{1}-\lambda_{2}\right)}\|f\|_{p_{1}}\|g\|_{p_{2}}\|K\|_{p_{0}}
$$

for every $\lambda_{1}, \lambda_{2} \in D$ and we want now to minimize. Since $D$ is a convex domain and $F(x, y)=\left(|\alpha||\alpha-\beta|^{-1}\right)^{-x}\left(|\beta||\alpha-\beta|^{-1}\right)^{-y}$ is a convex function in $D$, the minimal costant is attained in one of the three vertex of the triangle:

$$
\begin{aligned}
& \left(\max \left(p_{1}^{-1}-p_{3}^{-1}, 0\right), \max \left(p_{2}^{-1}-p_{3}^{-1}, 0\right)\right) \\
& \left(\max \left(p_{1}^{-1}-p_{3}^{-1}, 0\right), \min \left(p_{1}^{\prime-1}, p_{2}^{-1}, p_{3}^{\prime-1}, p_{0}^{\prime-1}\right)\right) \\
& \left(\min \left(p_{1}^{-1}, p_{2}^{\prime-1}, p_{3}^{\prime-1}, p_{0}^{\prime-1}\right), \max \left(p_{2}^{-1}-p_{3}^{-1}, 0\right)\right)
\end{aligned}
$$

that is

$$
\left\|H_{K, \alpha, \beta}(f, g)\right\|_{p_{3}} \leq C_{\alpha, \beta, p_{1}, p_{2}, p_{0}}\|f\|_{p_{1}}\|g\|_{p_{2}}\|K\|_{p_{0}}
$$

where $C_{\alpha, \beta, p_{1}, p_{2}, p_{0}}$ is the minimum of the three quantities:

$$
\begin{aligned}
& |\alpha|^{-\max \left(p_{1}^{-1}-p_{3}^{-1}, 0\right)}|\beta|^{-\max \left(p_{2}^{-1}-p_{3}^{-1}, 0\right)}|\alpha-\beta|^{-\min \left(p_{1}^{-1}, p_{2}^{-1}, p_{3}^{-1}, p_{0}^{\prime-1}\right)} \\
& |\alpha|^{-\max \left(p_{1}^{-1}-p_{3}^{-1}, 0\right)}|\beta|^{-\min \left({p_{1}^{\prime}}^{-1}, p_{2}^{-1}, p_{3}^{\prime-1}, p_{0}^{\prime-1}\right)}|\alpha-\beta|^{-\max \left(p_{2}^{-1}-p_{1}^{\prime-1}, 0\right)} \\
& |\alpha|^{-\min \left(p_{1}^{-1}, p_{2}^{\prime-1}, p_{3}^{\prime-1}, p_{0}^{\prime-1}\right)}|\beta|^{-\max \left(p_{2}^{-1}-p_{3}^{-1}, 0\right)}|\alpha-\beta|^{-\max \left(0, p_{1}^{-1}-p_{2}^{\prime-1}\right)}
\end{aligned}
$$


which, on the surface of the octahedron, are the same bounds we already had (in fact, the three bounds coincide in each face).

3.3. The third condition. The last result gives a sufficient condition of boundedness for bilinear multipliers. It gives a condition over the symbol of the operator instead of over the kernel.

Proposition 3.3. Let $m \in L^{q}\left(\mathbb{R}^{2}\right)$ with $1 \leq q \leq 4$. Then $m$ is $\left(p_{1}, p_{2}, p_{3}\right)$ multiplier for all exponents such that $1 \leq p_{1}, p_{2}, p_{3}^{\prime} \leq \min (2, q), q \notin\left\{p_{1}, p_{2}, p_{3}^{\prime}\right\}$ and $p_{1}^{-1}+p_{2}^{-1}+p_{3}^{\prime-1}=1+2 q^{-1}$. Moreover, $\|m\|_{\mathcal{M} B_{p_{1}, p_{2}, p_{3}}} \leq\|m\|_{q}$.

Proof. By duality it is enough to prove that for every $f, g, h \in \mathcal{S}$

$$
I=\left|\int_{\mathbb{R}^{2}} \hat{f}(\xi) \hat{g}(\eta) m(\xi, \eta) \hat{h}(-\xi-\eta) d \xi d \eta\right| \leq C_{m}\|f\|_{p_{1}}\|g\|_{p_{2}}\|h\|_{p_{3}^{\prime}}
$$

If $q=1$ then $I \leq\|m\|_{1}\|\hat{f}\|_{\infty}\|\hat{g}\|_{\infty}\|\hat{h}\|_{\infty} \leq\|m\|_{1}\|f\|_{1}\|g\|_{1}\|h\|_{1}$.

If $q>1$, we define $\tilde{p}=\left(\tilde{p}_{1}, \tilde{p}_{2}, \tilde{p}_{3}^{\prime}\right)$ by

$$
\tilde{p}_{1}=\frac{p_{1}(q-1)}{q-p_{1}} \quad \tilde{p}_{2}=\frac{p_{2}(q-1)}{q-p_{2}} \quad \tilde{p}_{3}^{\prime}=\frac{p_{3}^{\prime}(q-1)}{q-p_{3}^{\prime}}
$$

which satisfy:

$$
1 \leq \tilde{p}_{1}, \tilde{p}_{2}, \tilde{p}_{3}^{\prime} \leq \infty, \quad \tilde{p}_{i}^{\prime}=\frac{p_{i}^{\prime}}{q^{\prime}} \quad i=1,2, \quad \tilde{p}_{3}=\frac{p_{3}}{q^{\prime}}, \quad \frac{1}{\tilde{p}_{1}^{\prime}}+\frac{1}{\tilde{p}_{2}^{\prime}}+\frac{1}{\tilde{p}_{3}}=2
$$

Then, by Hölder, Young and Hausdorff-Young inequalities we have

$$
\begin{aligned}
& I \leq\|m\|_{q}\left(\int_{\mathbb{R}^{2}}|\hat{f}(\xi)|^{q^{\prime}}|\hat{g}(\eta)|^{q^{\prime}}|\hat{h}(-\xi-\eta)|^{q^{\prime}} d \xi d \eta\right)^{\frac{1}{q^{\prime}}}=\|m\|_{q}\left(|\hat{f}|^{q^{\prime}} *|\hat{g}|^{q^{\prime}} *|\hat{h}|^{q^{\prime}}\right)(0)^{\frac{1}{q^{\prime}}} \\
& \leq\|m\|_{q}\left\||\hat{f}|^{q^{\prime}} *|\hat{g}|^{q^{\prime}} *|\hat{h}|^{q^{\prime}}\right\|_{\infty}^{\frac{1}{q^{\prime}}} \leq\|m\|_{q}\left(\left\||\hat{f}| q^{q^{\prime}}\right\|_{\tilde{p}_{1}^{\prime}}\left\||\hat{g}|^{q^{\prime}}\right\|_{\tilde{p}_{2}^{\prime}}\left\||\hat{h}|^{q^{\prime}}\right\|_{\tilde{p}_{3}}\right)^{\frac{1}{q^{\prime}}} \\
& =\|m\|_{q}\|\hat{f}\|_{\tilde{p}_{1}^{\prime} q^{\prime}}\|\hat{g}\|_{\tilde{p}_{2}^{\prime} q^{\prime}}\|\hat{h}\|_{\tilde{p}_{3} q^{\prime}}=\|m\|_{q}\|\hat{f}\|_{p_{1}^{\prime}}\|\hat{g}\|_{p_{2}^{\prime}}\|\hat{h}\|_{p_{3}} \leq\|m\|_{q}\|f\|_{p_{1}}\|g\|_{p_{2}}\|h\|_{p_{3}^{\prime}}
\end{aligned}
$$

Remark 3.4. Though $K \in L^{p}$ for some $1<p \leq 2$ none of the functions $m(\xi, \eta)=\hat{K}(\alpha \xi+\beta \eta) \in L^{q}\left(\mathbb{R}^{2}\right)$ for $1 \leq q \leq 4$. So, this result is neither a generalization nor a particularization of proposition 3.2. 


\section{REFERENCES}

1. C. Bennet and R. Sharpley, Interpolation of operators, Pure and applied mathematics vol. 129, Academic Press, Inc., New York (1988).

2. Calderón A.P., Cauchy integrals on Lipschitz curves and related operators. Proc. Natl. Acad. Sci. USA, Vol. 74, pp. 1324-1327, [1977]. Zbl 0373.44003

3. Fefferman C., Pointwise convergence of Fourier series. Ann. Math. 98, pp. 551-571, [1973] Zbl 0268.42009

4. Gilbert J. and Nahmod A., Boundedness of bilinear operators with non-smooth symbols Math. Res. Lett. 7 [2000] pp. 767-778 Zbl 0987.42017

5. Gilbert J. and Nahmod A., Bilinear operators with non-smooth symbols J. Fourier Anal. Appl. 7 [2001] pp. 437-469. Zbl 0994.42014

6. Grafakos L. and Li X., Uniform bounds for the bilinear Hilbert transform I Annals of Mathematics 159 (2004), no. 3, 889-933. Zbl 1071.44004

7. M. Lacey On the bilinear Hilbert transform Doc. Math., vol. II, 647-656,[1998] Zbl 0963.42007

8. Lacey M. and Thiele C., $L^{p}$ bounds on the bilinear Hilbert transform for $2<p<\infty$ Ann. Math. 146, [1997], pp. 693-724. Zbl 0914.46034

9. Lacey M. and Thiele C., On Calderón's conjecture. Ann. Math. 149 no 2 [1999] pp. 475-496 Zbl 0934.42012

10. Lacey M., The bilinear maximal function maps into $L^{p}$ for $2 / 3<p \leq 1$, Ann. Math. (2) 151 [2000] no 1 pp. 35-57 Zbl 0967.47031

11. Larsen R., An introduction to the theory of multipliers, vol. 175, Springer-Verlag (1971) Zbl 0213.13301

12. Muscalu, C., Tao, T. and Thiele, C Multilinear operators given by singular multipliers J. Amer. Math. Soc. 15 [2002] pp. 469-496.

13. Muscalu C., Tao T. and Thiele C., Uniform estimates on multi-linear operators with modulation symmetry J. Anal. Math. 88, 255-309 (2002) Zbl 1041.42013

14. Thiele C., On the bilinear Hilbert transform. Universitat Kiel, Habilitation [1998].

Departament D'Anàlisi Matemàtica, Universitat de València, 46100 BurJASSOT, VALÈnCIA, SPAin

E-mail address: paco.villarroya@uv.es 